\title{
Types and Causalities in Dead Patients Due to Traumatic Injuries
}

\author{
Mahnaz Yadollahi ${ }^{1}$; Shahram Paydar ${ }^{1}$; Golnar Sabetianfard Jahromi ${ }^{2}$; Hosseinali Khalili ${ }^{3}$; \\ Samie Etemadi ${ }^{1}$; Hamidreza Abbasi ${ }^{1}$; Shahram Bolandparvaz ${ }^{1, *}$ \\ ${ }^{1}$ Trauma Research Center, Shahid Rajaee Trauma Hospital, Shiraz University of Medical Sciences, Shiraz, IR Iran \\ 2 Department of Anesthesiology, Faghihi Hospital, Shiraz University of Medical Sciences, Shiraz, IR Iran \\ 3 Department of Neurosurgery, Nemazee Teaching Hospital, School of Medicine, Shiraz University of Medical Sciences, Shiraz, IR Iran \\ ${ }^{*}$ Corresponding author: Shahram Bolandparvaz, Trauma Research Center, Shahid Rajaee Trauma Hospital, Shiraz University of Medical Sciences, Shiraz, IR Iran. Tel/Fax: +98- \\ 7116364001, E-mail: Shaboland@sums.ac.ir
}

Received: December 11, 2014; Revised: December 28, 2014; Accepted: February 3, 2015

\begin{abstract}
Background: Trauma constitutes a major public health problem in our country and contributes significantly to unacceptably high morbidity and mortality.

Objectives:This study aimed to evaluate the epidemiology of trauma in dead patients referred to Shahid Rajaee Trauma Hospital, Shiraz, Iran.

Patients and Methods: In a cross-sectional study, all patients with trauma admitted to our center were enrolled between March 2011 and February 2012. Age, gender, months of referring, causalities, and injured body parts of the victims were extracted from the data registry and analyzed.

Results: A total of 9113 patients, including 7163 (78.6\%) males and 1950 (21.4\%) females were evaluated. Among them, 479 patients (5.3\%) had died. There was a significant difference between the age of alive and dead patients ( $35.70 \pm 0.18$ and $45.44 \pm 1.01$ years, respectively ( $P$ $<0.001)$ ). In addition, dead men had significantly higher percentage than alive men ( $83.7 \%$ and $78.3 \%$, respectively, $\mathrm{P}=0.005)$. The highest percentages of men and women who referred to our center and died were in September. Crashing by car had the most frequency of dead (247 cases) in referred patients and motorcycle accident, stabbing, and falling related injuries with 67,36 and 26 dead cases were in the next ranks. Thorax injury had the most frequency between dead patients ( $53.2 \%$ of all dead) and the intracranial injury and trauma to extremities altogether were in the next ranks, which constituted more than $18.8 \%$ of all dead.

Conclusions: Our data demonstrated that car and motorcycle accidents are the most important cause of trauma and thorax trauma had the most frequency among dead patients. Epidemiological evaluations and preventive measures such as this study should be conducted to provide valuable data.
\end{abstract}

Keywords: Epidemiology; Accident; Mortality; Motorcycle; Trauma

\section{Background}

Trauma is one of the most important causes of mortality and morbidity worldwide, which can affect many parts of the body, including brain, extremities, and internal organs. It is the leading cause of losing potential years of productive life (1). In 2009, trauma is the sixth leading cause of death and the fifth leading cause of significant disability worldwide, resulting in five million or $10 \%$ of all deaths annually (2). Trauma, after cancer and cardiac diseases is the leading cause of premature deaths in people lower than 65 years old in many countries $(3,4)$. Also in Iran, injuries (as the first cause of losing years of life) have attracted the attention of health decision makers in recent years (5). Monitoring traumatic patients can help us to take information about types, grades, severity, incidence, prevalence, and other characteristics of trauma injuries. These data help government to control and prevent trauma injuries, also, build up new protocols of trauma management, facilitate functions of trauma centers and utilize resources and materials more efficiently
(1). There are several causes of inducing trauma injuries in the general population such as falling, crashing by motor vehicles, etc. In addition; the place of trauma injuries ranges from head and neck to extremities.

Although in recent years the number of road traffic fatalities in developed and developing countries such as Iran per road user has decreased (6), there is still a disproportionate number of fatalities attributed to vehicular trauma. Road traffic accident is considered as the second leading cause of death in Iran while trauma death rate per 100000 population in the country is 58 in comparison to the rate of 99 in the world (7-10). Additionally, it had been reported that road traffic injuries was the major source of injuries in patients presenting to the hospitals of some African countries such as Uganda, Ghana, and Tanzania (11). However, in another study from Sierra Leone, a mid-income West African country, fall, lacerations, and crush injuries were the first and second most common causes of injury, respectively (12). On the other

Copyright (C) 2015, Kashan University of Medical Sciences. This is an open-access article distributed under the terms of the Creative Commons Attribution-NonCommercial 4.0 International License (http://creativecommons.org/licenses/by-nc/4.0/) which permits copy and redistribute the material just in noncommercial usages, provided the original work is properly cited. 
hand, falls are among the life events that lead to injury and in serious cases disability or death (13).

In some of the previous epidemiological studies, the prevalence of trauma causalities was evaluated. In these studies, it had been demonstrated that fall was the leading cause of injury among the elderly population, while road traffic injuries being the leading cause among the younger group (14). Also, based on a report from WHO in 2004, between 100 and 300 per 100,000 adult patients per year are treated in hospitals for mild traumatic brain injury, worldwide (15). In another study from Tanzania in 2012, among all injuries, trauma to the head and extremities had the highest percentage(11). Environmental, physiological, and psychological factors may commonly contribute to the incidence of all types of accidents in healthy people, especially in fall accidents. While any single accident is in itself sporadic, finding trends in the frequency of such accidents throughout the year in patients of one city may be useful for planning the provision of health service facilities. Trauma may be classified by demographic characteristics such as age or gender. It may also be classified by the type of force applied to the body such as blunt trauma or penetrating trauma. Besides the trauma causalities, the place of trauma is very important in morbidity and mortality of specific trauma. Therefore, trends as described here emphasize the continuous epidemiological evaluation of all traumatic injuries and outcome. In a single center retrospective study, we collected detailed data on demography, injury patterns, causalities, and outcome of all traumatic injuries in Shiraz.

\section{Objectives}

This study aimed to evaluate the epidemiology of trauma in dead patients referred to Shahid Rajaee Trauma Hospital, Shiraz, Iran. The findings are compared with earlier single and multicenter series to detect support for some of the trends in epidemiology and outcomes suggested in the literature.

\section{Patients and Methods}

\subsection{Study Setting and Population}

With assuming that mortality rate is about $5 \%(\mathrm{P}=0.05$, $\alpha=0.05$, and $d=0.05$ ) and estimated population size of 1400000 people (16), a sample size of 384 patients was calculated. However, in this cross-sectional study, we included all trauma patients admitted to Shahid Rajaee Trauma Hospital (a level I referral trauma center for Fars Province, affiliated to Shiraz University of Medical Sciences, Shiraz, Iran), over a period of 12 months from March 2011 to February 2012. This hospital is a referral center of trauma for Fars Province, Iran. Shiraz is the capital and main city of Fars Province, Southern of Iran with an estimated population of 1.4 million according to the recent national census (16). We had no exclusion criteria for this study and all referred patients were included. At the time of arrival to the Emergency Department, all patients were visited and carefully examined by a general surgery resident and his or her evaluation was recorded in respected medical forms. Medical charts of the patients were reviewed and the data were collected by trained research staff supervised by a physician and were entered into stand-alone databases available at this center. Demographic variables, including age, gender, and reception date were collected from the medical records.

\subsection{Outcome Assessment}

Vital status (dead or alive), causalities, and place of injuries, including intracranial and neck, intra-thoracic, intra-abdominal, extremities, vascular hemorrhage, and others nonclassified traumas like neoplasm, unspecified multiple injuries and so on were extracted from medical files. Trauma causalities were classified into stabbing (penetration with a sharp or pointed object at close range), car accident (car crashed to other vehicles or car crashed to people), motorcycle accident (motorcycle crashed to other vehicles or motorcycle crashed to people), falling, gun shot, assault (blunt and traumatic), and electric shock. Other causes, which were not placed in these categories such as alcohol poisoning, explosion, animal bite and so on, were categorized as other causalities. Our main outcomes were sex and age distribution in death and discharge patients. Also, the frequency of specific causalities of trauma and place of injuries were evaluated based on sex and age of patients.

\subsection{Statistical Analysis}

Quantitative data were expressed as mean \pm standard deviation and qualitative ones as frequency and percentage. Data were analyzed using SPSS version 20.0 (SPSS Ltd., Working, Surrey, UK) for Windows. Mostly, descriptive data will be presented. Differences in gender and age dependent mortality rates were calculated using Pearson $\chi^{2}$ and independent sample t tests. A P $<0.05$ was considered significant.

\section{Results}

Overall, 9113 injured patients admitted to Shahid Rajaee Trauma Hospital wereenrolled in thisstudy.The frequency,

Table 1. Age, Gender, and Frequency Comparison Between Alive and Dead Referred Patients

\begin{tabular}{lccc}
\hline Variable & Alive & Dead & Pvalue \\
\hline Frequency, No. (\%) & $8634(94.7)$ & $479(5.3)$ & \\
Gender & & & 0.005 \\
Male & 6762 & 401 & \\
Female & 1872 & 78 & \\
Age, $^{\mathrm{a}}$ & $35.70 \pm 0.18$ & $45.44 \pm 1.01$ & $<0.001$ \\
\hline
\end{tabular}

a Data are presented as mean \pm SEM. 
Figure 1. Frequency and Percentage of Dead Patients Among 479 Patients Referred to the Shahid Rajaee Trauma Hospital from March 2011 to February 2012
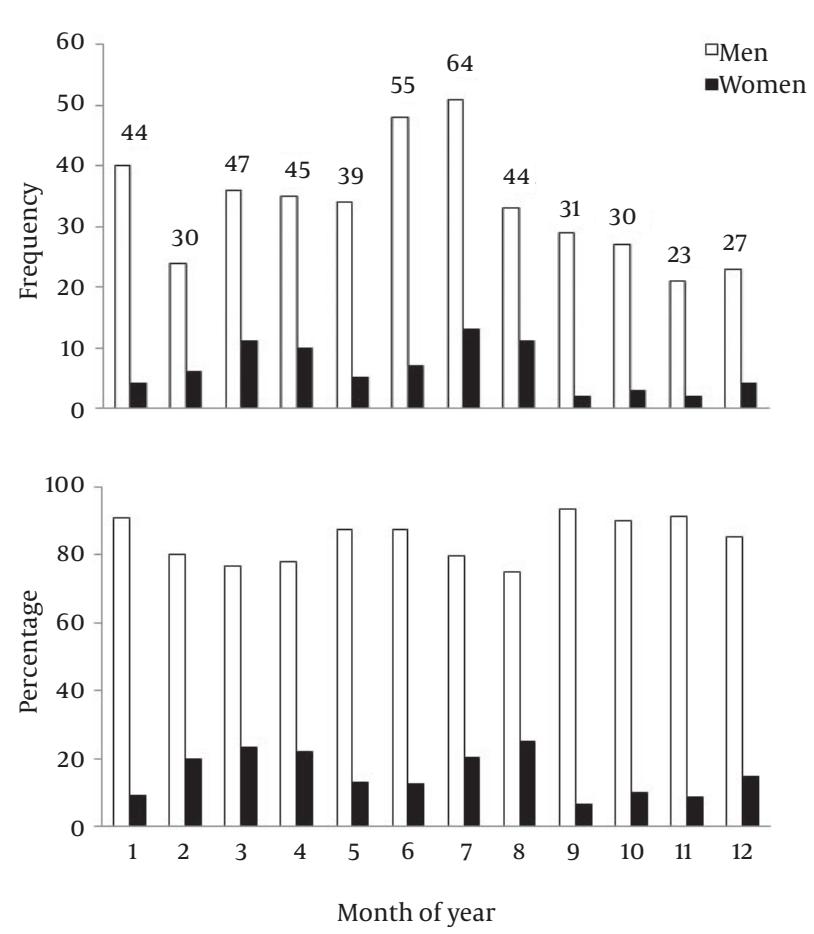

The number is started from March 1, 2011 to February 12, 2012.

Figure 2. Frequency and Percentage of Different Causalities of Trauma in Dead Patients Among 479 Patients Referred to the Shahid Rajaee Trauma Hospital from March 2011 to February 2012
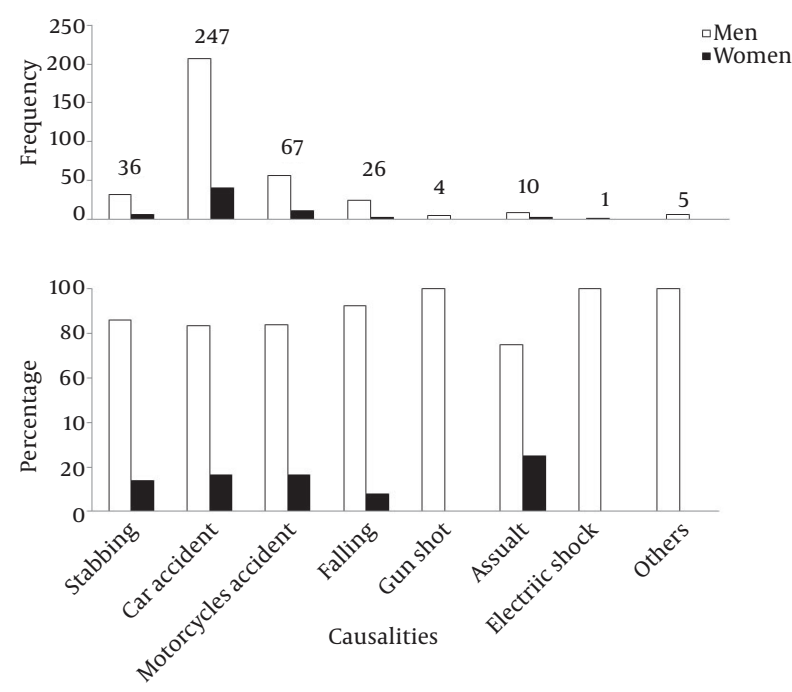

The number started from March 1, 2011 to February 12, 2012.
Figure 3. Frequency and Percentage of Different Types of Trauma in Dead Patients Among 479 Patients Referred to the Shahid Rajaee Trauma Hospital from March 2011 to February 2012
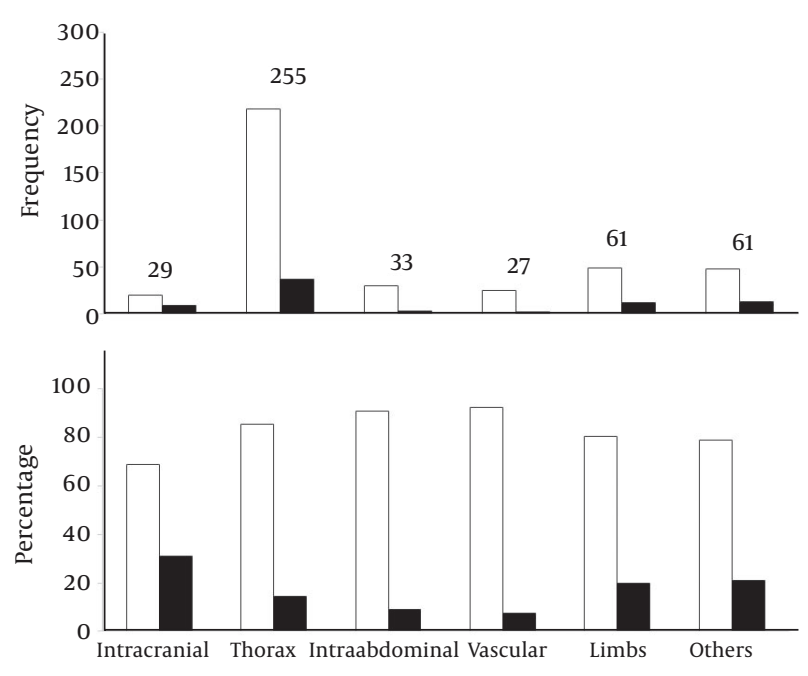

Type of Trauma

The number started from March 1, 2011 to February 12, 2012.

percentage, sex, and age of dead and alive referred patients and their statistical comparison are presented in Table 1. Vital status evaluation of all patients demonstrated that the death rate is $5.3 \%$. There was a significant difference between the mean \pm SEM of the age of alive and dead patients $(\mathrm{P}<0.001)$. The frequency of men and women in alive and dead patients was compared and our analysis demonstrated that, although the frequency of alive men was obviously higher than dead men, but dead men had significantly higher percentage than alive men $(P=0.005)$. Our next analysis was concerned on dead patients and demonstrated that there was no significant differences between age of men (45.48 \pm 1.12 years) and women ( 45.20 \pm 2.42 years $)(P=0.917)$. Frequency and percentage of men and women of dead patients in different months were presented in Figure 1. The highest number of traumatic patients were referred to our center during September (904 patients) and April (806 patients). As it is shown, the higher percentage of men and women, which referred to our respected center and died there, happened in September. In each trauma, the frequency and percentage of men and women are presented in Figure 2 and as shown, crashing by car had the highest dead rate (247 cases) and motorcycle accident, stabbing, and falling-related injuries with 67,36 , and 26 dead cases were in the next ranks. Finally, descriptive analysis of the contribution of each trauma in dead patient and gender classification in each type of trauma is presented in Figure 3. Our analysis demonstrated that thorax injury had the highest frequency among dead patients (53.2\% of all dead) and the intracranial injury and trauma to extremities altogether was in the next rank and constituted more than $18.8 \%$ of all dead. 


\section{Discussion}

The present study evaluated the epidemiological features and descriptively compared trauma injuries in patients referred to Shahid Rajaee Trauma Hospital over a 12-month period. Nine thousand one hundred and thirteen patients were referred to this center during this period, and among them 479 patients died. The majority of these dead patients were men, mostly referred in October with the main cause of car accident and thorax injuries. The male to female ratio was approximately $4: 1$ and this is also comparable to other related studies $(8,17,18)$. On the other hand, the mortality rate in this study (5.2\%) was in the range of previous report (5), but was approximately higher than some Iranian reports $(17,19)$.

Decrease in mortality and morbidity rates has been noticed in well-developed countries with a high economic income due to a prompt systematic trauma organization and their special services (20). Similar studies concerning the epidemiology of the trauma, its types, and severity in different areas as well as classification of the corresponding data have been conducted locally and worldwide. A prospective cross-sectional study, including all patients admitted to Nemazee Teaching Hospital from 2009 to 2010 demonstrated that most of the trauma injuries mostly occurred during summer and hot weather in Shiraz (1). Our results were partly in agreement with those results and demonstrated that the most of the traumatic patients were referred to our center during September and April. However, the most deaths in referred patients took place in September. In a retrospective review of patients with trauma admitted to the Acute and Emergency Care of an acute care hospital of Singapore between January 2011 and December 2012, it had been demonstrated that fall was the leading cause of injury among the elderly population, while road traffic injuries were the leading cause among the younger group (14). Although, in some previous studies, it has been reported that falling had the second (20) and sometimes the first (21) place of frequency among trauma causes, in our study, it had the third and fourth place of injury mechanism among all alive and dead patients.

Falling may happen at any age, but children and the aged people are at higher risk (22); therefore, preventive measures should be taken such as increasing the awareness of these groups about the seriousness of fall incidence and encouraging the aged individuals to get involved in fitness programs to remain physically fit and healthy (13). In a retrospective analysis from Scotland in 2012, it had been reported that the elderly population were a consistent proportion of the overall trauma population during the study period, but had a higher absolute mortality rate following trauma at $9.9 \%$ compared to the younger population who had a mortality rate of $4.4 \%$ (23). Also, in another retrospective analysis on all patients $>65$ years admitted to Queensland Trauma Registry (QTR) in Australia from 2003 to 2006, it had been concluded that older adults with severe injuries are at risk of poor outcomes (24). In line with these studies, the results of this study demonstrated that the age of traumatic patients who referred to our center and died was significantly higher compared to survived traumatic patients. Therefore, older patients with traumatic injuries had poor outcome and more susceptible to die after trauma.

Our results demonstrated that the main causes of death in traumatic patients, which referred to our trauma center was motorcycle traffic trauma. In recent years, there has been a steady increase in the number of road traffic injuries, and data for the 6-year-period (1995-2000) indicates that there has been an estimated $8 \%$ annual increase in the mortality rate due to these injuries (8). Motor vehicle accidents are among the ten leading causes of death and disability worldwide and have emerged as a serious public health concern (25). Furthermore, road traffic accident deaths are predicted to increase from 1.2 million in 2002 to 2.1 million in 2030 , primarily due to increased motor vehicle fatalities associated with economic growth in low- and middle-income countries (25). The above-mentioned report belonged to the year of 2006 and its prediction seems to be true as our results demonstrated. Other evaluations such as reports from Iran (20), Singapore (26), Tanzania (27), and Spain (28) also confirmed that motor vehicle accidents were the most common mechanism of trauma. A prospective cross-sectional study which comprised all consequently admitted patients to the Emergency Department from December 2003 through July 2004 was performed in Mashhad, North East of Iran. Their data demonstrated that most of the traumatized patients were male. Motor vehicle accidents and falling were considered as the most common mechanisms. Also, the head and neck injuries were the most common traumatized anatomic areas leading to their related morbidity and mortality (20). With a different manner of evaluation, Chalya et al. (27) reported that motorcycle was responsible for the majority of road traffic crashes (near 60\%). Furthermore, musculoskeletal (60.5\%) and head (52.1\%) were the most common body regions injured in Tanzania.

Our study had two major limitations. First, the duration of the study is fairly low. Second, there are some other emergency centers in Shiraz such as Nemazee Teaching Hospital and Faghihi Hospital emergency centers. Some of the traumatic patients may be referred to these centers, which interfere with our analysis. It is highly recommended to evaluate other traumatic patients referred to such centers to have a conclusive data to analyze. Furthermore, analysis in a long-time duration (may be 10 years) is seriously advised.

In conclusion, we found that the majority of dead patients were men, mostly referred in October with the main cause of car accident with thorax injuries. The patients who referred to our hospital can be considered 
Yadollahi M et al.

as a sample of the total population of Fars Province and provincial and national decisions can be made based on these results for prevention of trauma and decline in trauma related death. For every death, it is estimated that there are multiple hospitalizations, emergency department visits, and doctors' appointments. Also, the results of this descriptive study can be helpful for conducting operational and strategic decisions to reduce injury and death costs. However, further studies in other provinces of Iran and performing other national retrospective studies are highly recommended for finding more accurate and more comprehensive results. Similar researches in distinct age and sex groups are also needed.

\section{Acknowledgements}

The authors kindly appreciate the staff of the Trauma Center of Shahid Rajaee Trauma Hospital, Shiraz, Iran.

\section{Authors' Contributions}

Study concept: Mahnaz Yadollahi, Shahram Paydar, Golnar Sabetianfard Jahromi, Hosseinali Khalili, Samie Etemadi, Hamidreza Abbasi, and Shahram Bolandparvaz. Writing the article: Mahnaz Yadollahi, Shahram Paydar, Golnar Sabetianfard Jahromi, and Hamidreza Abbasi. Data obtaining and statistical analysis: Shahram Paydar, Mahnaz Yadollahi, and Hamidreza Abbasi. Revising the draft: Golnar Sabetianfard Jahromi, Hosseinali Khalili, Samie Etemadi, Shahram Bolandparvaz.

\section{References}

1. Abbasi HR, Mousavi SM, Taheri AA, Niakan MH, Bolandparvaz S, Paydar S. Pattern of traumatic injuries and Injury Severity Score in a major trauma center in Shiraz, Southern Iran. Bull Emerg Trauma. 2013;1(2):81-5.

2. Soreide K. Epidemiology of major trauma. Br J Surg. 2009; 96(7):697-8.

3. Paydar S, Bazrafkan H, Golestani N, Roozbeh J, Akrami A, Moradi $\mathrm{AM}$. Effects of Intravenous Fluid Therapy on Clinical and Biochemical Parameters of Trauma Patients. Emergency. 2014;2(2):pp. 90-5.

4. Yoon PW, Bastian B, Anderson RN, Collins JL, Jaffe HW, Centers for Disease C, et al. Potentially preventable deaths from the five leading causes of death--United States, 2008-2010. MMWR Morb Mortal Wkly Rep. 2014;63(17):369-74.

5. Zargar M, Kalantar Motamedi SM, Karbakhsh M, Ghodsi SM, Rahimi-Movaghar V, Panahi F, et al. Trauma care system in Iran. Chin J Traumatol. 2011;14(3):131-6.

6. Heyes GJ, Craig J, Hinds JD, Kealey DW. The burden of motorcycle trauma and seasonal change at a regional trauma centre. Ulster Med J. 2014;83(1):55-6.

7. Haghparast-Bidgoli H, Khankeh H, Johansson E, Yarmohammadian MH, Hasselberg M. Exploring the provision of hospital trauma care for road traffic injury victims in Iran: a qualitative approach. J Inj Violence Res. 2013;5(1):28-37.
8. Montazeri A. Road-traffic-related mortality in Iran: a descriptive study. Public health. 2004;118(2):110-3.

9. Shams M, Rahimi-Movaghar V. Risky driving behaviors in Tehran, Iran. Traffic Inj Prev. 2009;10(1):91-4.

10. Vahdati SS, GhafarZad A, Rahmani F, Panahi F, Rad AO. Patterns of road traffic accidents in north west of Iran during $2013 \mathrm{New}$ Year Holidays: complications and casualties. Bull Emerg Trauma. 2014;2(2):82-5.

11. Casey ER, Muro F, Thielman NM, Maya E, Ossmann EW, Hocker $\mathrm{MB}$, et al. Analysis of traumatic injuries presenting to a referral hospital emergency department in Moshi, Tanzania. Int J Emerg Med. 2012;5(1):28.

12. Stewart KA, Groen RS, Kamara TB, Farahzad MM, Samai M, Cassidy LD, et al. Traumatic injuries in developing countries: report from a nationwide cross-sectional survey of Sierra Leone. JAMA Surg. 2013;148(5):463-9.

13. Sayyah M, Khosravi G, Bigdeli M. Frequency of fall-related injuries of female patients referred to the trauma center in the city of Kashan from years 2005 to 2008. Chin JTraumatol.2013;16(1):46-50.

14. Wui LW, Shaun GE, Ramalingam G, Wai KM. Epidemiology of trauma in an acute care hospital in Singapore. J Emerg Trauma Shock. 2014;7(3):174-9.

15. Cassidy JD, Carroll LJ, Peloso PM, Borg J, von Holst H, Holm L, et al. Incidence, risk factors and prevention of mild traumatic brain injury: results of the WHO Collaborating Centre Task Force on Mild Traumatic Brain Injury.J Rehabil Med.2004(43 Suppl):28-60.

16. Vakili V, Danaei M, Abdollahifard G, Askarian M. Predictors of screening programs adherence: Report of a population based study in Shiraz, Iran. Int J Med Sci Public Health. 2014;3(9):1161-8.

17. Fazel MR, Fakharian E, Mahdian M, Mohammadzadeh M, Salehfard L, Ramezani M. Demographic Profiles of Adult Trauma During a 5 Year Period (2007-2011) in Kashan, IR Iran. Arch Trauma Res. 2012;1(2):63-6.

18. Zargar M, Modaghegh MH, Rezaishiraz H. Urban injuries in Tehran: demography of trauma patients and evaluation of trauma care. Injury. 2001;32(8):613-7.

19. Rasouli MR, Saadat S, Haddadi M, Gooya MM, Afsari M, RahimiMovaghar V. Epidemiology of injuries and poisonings in emergency departments in Iran. Public Health. 2011;125(10):727-33.

20. Modaghegh MH, Saremi E, Mohamadian M, Jafarzadeh R. Characteristics of trauma in North East Iran and the prevention strategies. Arch Iran Med. 2013;16(10):576-9.

21. Sauter C, Zhu S, Allen S, Hargarten S, Layde PM. Increased risk of death or disability in unhelmeted Wisconsin motorcyclists. WMJ. 2005;104(2):39-44.

22. Mahdian M. Fall injuries: an important preventable cause of trauma. Arch Trauma Res. 2013;2(3):101-2.

23. O'Neill S, Brady RR, Kerssens JJ, Parks RW. Mortality associated with traumatic injuries in the elderly: a population based study. Arch Gerontol Geriatr. 2012;54(3):e426-30.

24. Aitken LM, Burmeister E, Lang J, Chaboyer W, Richmond TS. Characteristics and outcomes of injured older adults after hospital admission. J Am Geriatr Soc. 2010;58(3):442-9.

25. Mathers CD, Loncar D. Projections of global mortality and burden of disease from 2002 to 2030. PLoS Med. 2006;3(11).

26. Leong M, Mujumdar S, Raman L, Lim YH, Chao TC, Anantharaman V. Injury related deaths in Singapore. Hong Kong J Emerg Med. 2003;10:88-96.

27. Chalya PL, Mabula JB, Dass RM, Mbelenge N, Ngayomela IH, Chandika $\mathrm{AB}$, et al. Injury characteristics and outcome of road traffic crash victims at Bugando Medical Centre in Northwestern Tanzania. J Trauma Manag Outcomes. 2012;6(1):1.

28. Ferrando J, Rodriguez-Sanz M, Borrell C, Martinez V, Plasencia A. Individual and contextual effects in injury morbidity in Barcelona (Spain). Accid Anal Prev. 20 05;37(1):85-92. 